\title{
Management de la qualité dans la filière ananas en Martinique
}

Frédérique SAUDUBRAY ${ }^{a}$, Jean-Pierre HORRY ${ }^{b}$, Paul-Alex MARIE-ALPHONSINE ${ }^{b}$, David Cros $^{b}$, Alain SOLER ${ }^{b}$ *

a Pôle de Recherche Agronomique de la Martinique (PRAM), Cemagref, BP 214, 97285 Lamentin Cedex 2, Martinique, France

b Pôle de Recherche Agronomique de la Martinique (PRAM), Cirad-Flhor, BP 214, 97285 Lamentin Cedex 2 , Martinique, France alain.soler@cirad.fr

\section{Quality management in the pineapple industry of Martinique.}

Abstract - Introduction. The pineapple industry in Martinique is implementing a significant reorganization, as already achieved in other food sectors, to comply with new requirements for food security and environmental protection: quality management at all levels of the industry and introduction of new pineapple varieties. Based on the analysis of the current practices, needs for changes and tools for the implementation of a new production system are proposed by the authors. Materials and methods. A typology of the farms is proposed after an analysis of the practices and the strategies of the farmers. A steering committee, including most stakeholder representatives, builds a system of quality management based on the Agriconfiance ${ }^{\circledR}$ norms. New pineapple varieties are evaluated both for processing and the fresh market. Results and discussion. Gaps between recommendations and actual practices are very variable but generally large: there are too many inputs, either pesticides or fertilizers or both. The system of management is based on a contract between the association and the producer. The rules are described in two codes of practice, one for fruit production in the fields, the other for fruit processing. A traceability system has been implemented, allowing records and forecasts at plot level. New pineapple varieties have also been tested in the processing plant of the association, with better results than the old variety Smooth Cayenne. Conclusion. The reorganization of the pineapple industry in Martinique results from economical considerations. Its implementation required the development of a global system for the quality management and a traceability system at plot level to ensure food security. Reducing the variability of agricultural practices and getting closer to the recommendations will increase the quality of the production. To do so, a guide book for good practices will be elaborated. The new pineapple varieties allowing better yield after processing and quality of product, fresh or processed, will also be determinant for a successful reorganization of the industry.

Martinique / Ananas comosus / crop management / food security / environmental protection / variety trials / fruits / quality

\section{Management de la qualité dans la filière ananas en Martinique.}

Résumé - Introduction. À l'instar d'autres secteurs de produits destinés à l'alimentation humaine, une restructuration profonde de la filière ananas martiniquaise s'accomplit pour répondre à de nouvelles exigences de sécurité alimentaire et de protection de l'environnement : management de la qualité sur l'ensemble de la filière et exploitation de nouvelles variétés d'ananas. À partir de l'analyse des pratiques actuelles, les auteurs ont évalué les besoins de changements, puis proposé quelques outils pour la mise en œuvre d'un système de production. Matériel et méthodes. Des enquêtes ont permis de connaître les pratiques et stratégies actuelles des producteurs d'ananas et d'établir une typologie des exploitations. Un comité de pilotage associant des représentants de l'ensemble de la filière élabore le système de management de la qualité en se basant sur la norme Agriconfiance ${ }^{\circledR}$. De nouvelles variétés sont en cours d'évaluation pour couvrir au mieux les besoins en ananas de qualité pour la transformation ou pour la vente en frais. Résultats et discussion. Les écarts constatés entre recommandations et pratiques culturales sont apparus très variables mais souvent importants : trop d'intrants en général, soit les pesticides, soit les engrais, soit les deux. Le système de management appliqué repose sur un contrat entre une coopérative et les producteurs. Les règles de qualité sont fixées dans deux cahiers des charges, l'un concernant la qualité de la production au champ, l'autre

* Correspondance et tirés à part la qualité du fruit pour l'usine. Un système de traçabilité à l'échelle de la parcelle a été élaboré. Il permet la prévision et l'enregistrement de toute activité liée à la production, avec la parcelle comme unité de base. Enfin, de nouvelles variétés hybrides sélectionnées par le Cirad ont pu être testées dans le nouveau processus de transformation de la coopérative, donnant de meilleurs résultats que l'ancienne variété Cayenne lisse. Conclusion. La restructuration de la filière ananas en Martinique résulte avant tout de considérations économiques. Cette démarche a conduit au développement d'un programme global d'assurance qualité, avec instauration d'un système de traçabilité parcellaire, destiné à garantir la sécurité alimentaire. Une homogénéisation des pratiques culturales est nécessaire, en se rapprochant des recommandations pour améliorer la qualité. Elles pourraient être rassemblées dans un guide de culture spécifique. L'adoption de nouvelles variétés d'ananas permettant d'obtenir de meilleurs rendements de transformation ainsi qu'une qualité accrue du produit frais ou transformé sera aussi un élément déterminant du succès de la démarche.

Martinique / Ananas comosus / conduite de la culture / sécurité alimentaire / protection de l'environnement / essai de variété / fruits / qualité
Fruits, 2006, vol. 61, p. 39-53 (c) 2006 Cirad/EDP Sciences All rights reserved DOI: 10.1051/fruits:2006004

RESUMEN ESPAÑOL, p. 53 


\section{Introduction}

Sécurité alimentaire, protection de l'environnement, traçabilité des produits, qualité accrue sont des problématiques qui préoccupent actuellement les producteurs d'ananas de Martinique. À l'instar d'autres secteurs de produits destinés à l'alimentation humaine, une restructuration profonde de la filière ananas martiniquaise est en cours d'accomplissement pour répondre à ces nouveaux engagements de la profession vis-àvis des consommateurs. Tout le secteur ananas se reconstruit autour de ces objectifs : l'usine de transformation Socomor ${ }^{1}$ propose de nouveaux produits (crush et cubes aseptiques à la place des tranches traditionnelles) et adopte un système d'assurance qualité basé sur les norme ISO 9002. Les exploitants diversifient leur production suivant la destination des récoltes (usine ou frais) et cherchent à modifier leurs pratiques en lien avec la nouvelle politique contractuelle développée par l'État [contrat territorial d'exploitation (CTE), puis contrat d'agriculture durable $(\mathrm{CAD})$ ] et à mettre en œuvre la norme Agriconfiance ${ }^{\circledR}[1]$.

La recherche agronomique accompagne cette restructuration en aidant la filière ananas sur trois aspects de la démarche entreprise :

- en recueillant et en analysant les informations concernant les pratiques et stratégies des producteurs, en tant qu'individus, afin de mieux cerner les domaines où des efforts particuliers doivent être réalisés et d'être sûr que la démarche entreprise cadrera au mieux avec leurs objectifs,

- en participant, au sein d'un comité de pilotage, à l'élaboration du système de management de la qualité (création de cahiers des charges et de systèmes de traçabilité parcellaire),

\footnotetext{
${ }^{1}$ La Socomor est une société coopérative agricole localisée à Morne Rouge, Martinique. C'est l'unique organisation de producteurs d'ananas de la Martinique et elle bénéficie, à ce titre, depuis 1976, d'un soutien communautaire à la production d'ananas transformés.
}

- en mettant au point de nouvelles variétés mieux adaptées aux marchés et aux nouveaux processus de transformation, destinées à améliorer la compétitivité de la filière grâce à une reconversion variétale partielle.

\section{Matériel et méthode}

\subsection{Analyse des pratiques et stratégies des producteurs}

La méthodologie utilisée a été basée sur les travaux de Capillon et Sébillote [2] pour définir une typologie à partir de l'étude des systèmes de production des exploitations. Par ailleurs, la définition et la mise en œuvre d'outils pour une normalisation (Agriconfiance ${ }^{\circledR}$, CAD, etc.) a nécessité de mieux connaître les pratiques des agriculteurs, ces dernières étant " par essence même, le reflet de la diversité des situations et des projets des agriculteurs " [3-5]. En effet, la connaissance et la compréhension des pratiques, des stratégies et des modalités de prise de décision des agriculteurs sont indispensables pour mesurer l'acceptabilité sociale, technique, économique et financière des mesures qui pourront être prises.

Des informations ont été recueillies grâce à des entretiens semi-directifs conduits auprès de la moitié des producteurs d'ananas martiniquais [6]. Lorsque cela a été possible, les déclarations des agriculteurs ont été confrontées avec des observations réalisées sur l'exploitation. En effet, selon Milleville [7], " la confrontation systématique du 'dit' et du 'fait' se révèle à l'expérience un moyen assez pertinent pour éclairer les raisons des choix techniques. S'exprime alors l'intervention de contraintes multiples qui expliquent que le 'réalisé' diffère du 'souhaitable' ou du 'prévu' ".

Le niveau de précision des informations recherchées a été déterminé selon les concepts d' "ignorance optimale " et de " degré acceptable d'imprécision" [8] :

- Le " degré d'ignorance optimale " résume le principe selon lequel il est fait le meilleur usage du temps disponible, l'attention étant portée sur ce qui est important et ce qui l'est 
moins étant négligé. Cela revient implicitement à ignorer une partie des informations potentiellement disponibles, en admettant qu'il n'est pas nécessaire de tout savoir.

- Le concept de " degré acceptable d'imprécision " recommande d'apprécier le niveau de détail nécessaire ou l'ordre de grandeur de la précision des informations jugées utiles.

Ainsi, notre objectif étant de préparer la mise en place d'une politique de production de fruits de qualité et de mesures de protection de l'environnement, les pratiques plus particulièrement étudiées ont porté sur les modalités de traitements phytosanitaires (défense des cultures, lutte contre les adventices), de fertilisation et de travail du sol (perspective de lutte contre l'érosion). Cependant, même si nous avons analysé les " manières de faire " et leur justification pour ces diverses pratiques, nous avons dû les situer au sein de l'ensemble de l'itinéraire technique pour comprendre la stratégie du producteur d'ananas. Il nous a donc fallu également caractériser cet itinéraire technique.

Pour analyser les données recueillies, l'itinéraire technique appliqué par chaque agriculteur a été découpé en une succession d'opérations techniques. Les opérations techniques ont été regroupées par thème: fertilisation, traitement phytosanitaire, etc. Chacun de ces thèmes a donné lieu à une analyse descriptive, à une comparaison des pratiques constatées avec les techniques prescrites, ainsi qu'à une analyse comparative de la manière de faire des différents planteurs ; il a été alors possible de regrouper et/ou de différencier les exploitations par une analyse factorielle des correspondances multiples.

\subsection{Management de la qualité des activités agricoles}

Un comité de pilotage "qualité " a été mis en place ; il comprend des producteurs, des chercheurs et du personnel d'encadrement de la Chambre d'agriculture en plus des cadres et techniciens de l'usine de transformation ; celle-ci met aussi en ouvre, par ailleurs, sa propre démarche interne basée sur la certification ISO.
Ce comité est assisté par des consultants spécialistes de la mise en ouvre de telles démarches en milieu agricole. Il a pour tâche de réaliser une "analyse de maîtrise des dangers " et de créer une base documentaire pour un système de management de la qualité en y intégrant les spécificités de la culture de l'ananas en Martinique, milieu insulaire de très petite taille caractérisé par son état de territoire de l'Union européenne et donc soumis aux changements radicaux de la réglementation concernant les produits phytosanitaires. Enfin, le comité doit proposer les mesures d'appui aux producteurs, notamment de formation, et le calendrier pour la mise en place du système.

\subsection{Base de données "producteurs " pour la traçabilité parcellaire}

\subsubsection{Programmation informatique}

La base de données " producteurs " est gérée par le logiciel Access de Microsoft.

\subsubsection{Base de données "producteurs"}

La base de données " producteurs " contient des éléments essentiels permettant le suivi des opérations entrant dans la définition de l'itinéraire technique :

- coordonnées géographiques de chaque exploitation,

- inventaire du parcellaire de chaque producteur en relation avec le système d'information géographique (SIG) de la Socomor,

- gestion des parcelles incluant toutes les données relatives aux activités agricoles qu'elles soient prévues ou réellement effectuées,

- formulaire de paramétrage de l'itinéraire technique permettant d'adapter cet itinéraire à la situation de chaque producteur en fonction des recommandations officielles,

- prévision et enregistrement de récoltes (couplage avec le logiciel de pesée de la Socomor),

- enregistrement des températures en continu sur les sites représentatifs des zones de production pour la prévision de récolte, 
Tableau I.

Fertilisation pratiquée par les agriculteurs par rapport aux préconisations.

\begin{tabular}{|c|c|c|}
\hline $\begin{array}{l}\text { Modalités } \\
\text { de fertilisation }\end{array}$ & $\begin{array}{l}\text { Préconisation de la } \\
\text { Chambre d'agriculture pour la } \\
\text { variété Cayenne lisse }\end{array}$ & Pratiques des agriculteurs \\
\hline Apport d'engrais liquide & $\begin{array}{l}\text { Pulvérisation d'urée et de sulfate de } \\
\text { potasse tous les mois à partir d'un mois } \\
\text { après plantation et jusqu'à un mois } \\
\text { avant le TIF } \\
\text { Augmentation progressive des doses }\end{array}$ & $\begin{array}{l}\text { Tous les agriculteurs pulvérisent de l'urée et du sulfate } \\
\text { de potasse et respectent les dates d'application } \\
27 \% \text { augmentent progressivement les doses } \\
37 \% \text { appliquent des doses constantes } \\
37 \% \text { « hésitent » entre les deux pratiques }\end{array}$ \\
\hline Apport d'engrais solide & $\begin{array}{l}\text { Trois apports d'un engrais } \\
12-4-24+4 \\
(500 \mathrm{~kg}, 1100 \mathrm{~kg}, 1100 \mathrm{~kg})\end{array}$ & 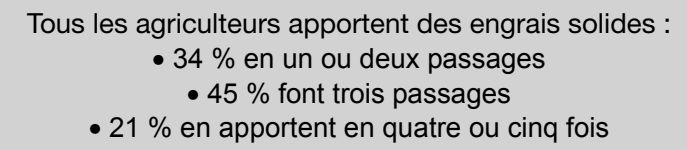 \\
\hline $\begin{array}{l}\text { Apports moyens d'azote }(\mathrm{N}) \\
\text { en } 1^{\mathrm{er}} \text { cycle }\end{array}$ & $\begin{array}{l}600 \mathrm{~kg} \text { en } 1^{\mathrm{er}} \text { cycle } \\
\text { (soit } 10 \mathrm{~g} \text { par plant par cycle) }\end{array}$ & $\begin{array}{l}\text { Moyenne par exploitation : } 824 \mathrm{~kg} \\
\text { Moyenne pondérée par ha : } 688 \mathrm{~kg}\end{array}$ \\
\hline $\begin{array}{l}\text { Apports moyens de potasse } \\
\left(\mathrm{K}_{2} \mathrm{O}\right) \text { en } 1^{\mathrm{er}} \text { cycle }\end{array}$ & $\begin{array}{c}1104 \mathrm{~kg} \text { en } 1^{\mathrm{er}} \text { cycle } \\
\text { (soit } 18,4 \mathrm{~g} \text { par plant par cycle) }\end{array}$ & $\begin{array}{l}\text { Moyenne par exploitation : } 1296 \mathrm{~kg} \\
\text { Moyenne pondérée par ha : } 1144 \mathrm{~kg}\end{array}$ \\
\hline Rapport $\left[\mathrm{K}_{2} \mathrm{O} / \mathrm{N}\right]$ & $\begin{array}{c}1,84 \\
\text { Entre } 2 \text { et } 2,5 \text { selon les ouvrages }\end{array}$ & $\begin{array}{c}\text { Moyenne }=1,66 \\
73 \% \text { des exploitations ont un rapport } \mathrm{K}_{2} \mathrm{O} / \mathrm{N}<1,80 \\
17 \% \text { respectent les prescriptions } \\
\mathrm{K}_{2} \mathrm{O} / \mathrm{N} \text { total }=0,5324 \times \mathrm{K}_{2} \mathrm{O} / \mathrm{N} \text { pulvérisation }+0,9414 \\
\left(\text { avec } R^{2}=0,7886\right)\end{array}$ \\
\hline
\end{tabular}

- calcul des flux prévisionnels de matière première (fruits) entrant à l'usine,

- calcul des besoins en intrants pour l'ensemble de la coopérative.

\subsubsection{Module de prévision de récolte}

Un module de prévision de récolte basé sur le modèle "Aloha pineapple " $([9,10]$ et Touron J., Fournier P., commun. pers., 2000) permet de prévoir les dates de récolte des ananas en utilisant les sommes de température calculées à partir des relevés quotidiens de températures ; ces relevés permettent en effet d'évaluer, pour une zone donnée, l'intervalle entre la date du traitement d'induction florale (TIF) des plants d'ananas et la récolte des fruits.

\subsection{Nouvelles variétés}

En complément de l'accompagnement de la démarche décrite précédemment, de nouvelles variétés, aptes à couvrir les besoins en ananas de qualité pour la transformation et la vente en frais, pourraient être proposées. Ces variétés sont issues d'un programme d'hybridation classique entre la variété
Cayenne lisse et la variété Péroléra. Cayenne lisse est la variété la plus répandue sur les marchés ; elle a de bonnes caractéristiques agronomiques et organoleptiques. La variété Péroléra a des caractéristiques de couleur particulièrement attrayantes (peau rouge et chair d'un jaune profond). Un certain nombre d'hybrides "Cayenne $\times$ Péroléra " intéressants ont été sélectionnés. Le principal hybride proposé actuellement aux producteurs pour une reconversion variétale partielle a été nommé "Flhoran 41 ". Cet hybride est en cours de test pour déterminer son potentiel agronomique et commercial en frais ou en transformation ; pour cette transformation, il fait l'objet de tests grandeur nature en usine avec le système de fabrication de cubes et crush aseptique.

\section{Résultats et discussion}

\subsection{Analyses des pratiques}

\subsubsection{Exemple de la fertilisation}

À partir d'entretiens avec les producteurs, il a été possible de proposer un récapitulatif 
des pratiques de fertilisation appliquées en premier cycle de culture (tableau I). Il en ressort que les planteurs d'ananas fertilisent, en azote et en potasse, beaucoup plus que nécessaire: l'apport moyen en azote est supérieur de $37,5 \%$ à la quantité préconisée par la Chambre d'agriculture et l'apport moyen en potasse est supérieur de 17,5\% à la quantité conseillée (figure 1). Seuls $30 \%$ des agriculteurs appliquent des quantités proches de celles préconisées. À l'opposé, plus de $10 \%$ d'entre eux appliquent plus du double des doses prescrites.

Pour une bonne croissance de la plante et des fruits, le rapport $\left[\mathrm{K}_{2} \mathrm{O} / \mathrm{N}\right]$ doit être compris entre 2 et 2,5 selon les recommandations techniques. Dans l'ensemble, les planteurs d'ananas de la Martinique appliquent, en raison d'apports d'azote excessifs, une fertilisation présentant un rapport $\left[\mathrm{K}_{2} \mathrm{O} / \mathrm{N}\right]$ trop faible ; en effet, pour $73 \%$ des itinéraires techniques le rapport $\left[\mathrm{K}_{2} \mathrm{O} /\right.$ $\mathrm{N}$ ] est inférieur à 1,80, le rapport moyen de $\left[\mathrm{K}_{2} \mathrm{O} / \mathrm{N}\right]$ étant 1,63. Parmi les $17 \%$ d'itinéraires techniques en adéquation avec les prescriptions techniques, il y a tous ceux suivis par les grandes exploitations.

En conclusion, $1 / 3$ des producteurs d'ananas de la Martinique appliquent des doses d'engrais nettement supérieures aux techniques prescrites. Cette pratique de surfertilisation concerne $25 \%$ de la surface plantée en ananas.

Près des 2/3 des agriculteurs, qui occupent cependant uniquement $1 / 3$ de la surface cultivée en ananas en Martinique, ne respectent pas l'équilibre préconisé entre les apports de potasse et ceux d'azote (rapport $\left[\mathrm{K}_{2} \mathrm{O} / \mathrm{N}\right]$ appliqué en pulvérisation trop faible).

\subsubsection{Exemple des pratiques de désherbage}

Le désherbage est avant tout chimique et variablement raisonné (figure 2).

Les planteurs d'ananas utilisent les herbicides " en généralisé " de deux manières distinctes : raisonnée ou systématique.

- Pour un désherbage chimique généralisé effectué de façon " raisonnée ", ils adaptent l'emploi d'herbicides à la quantité d'herbe à détruire ou en fonction du temps qu'ils

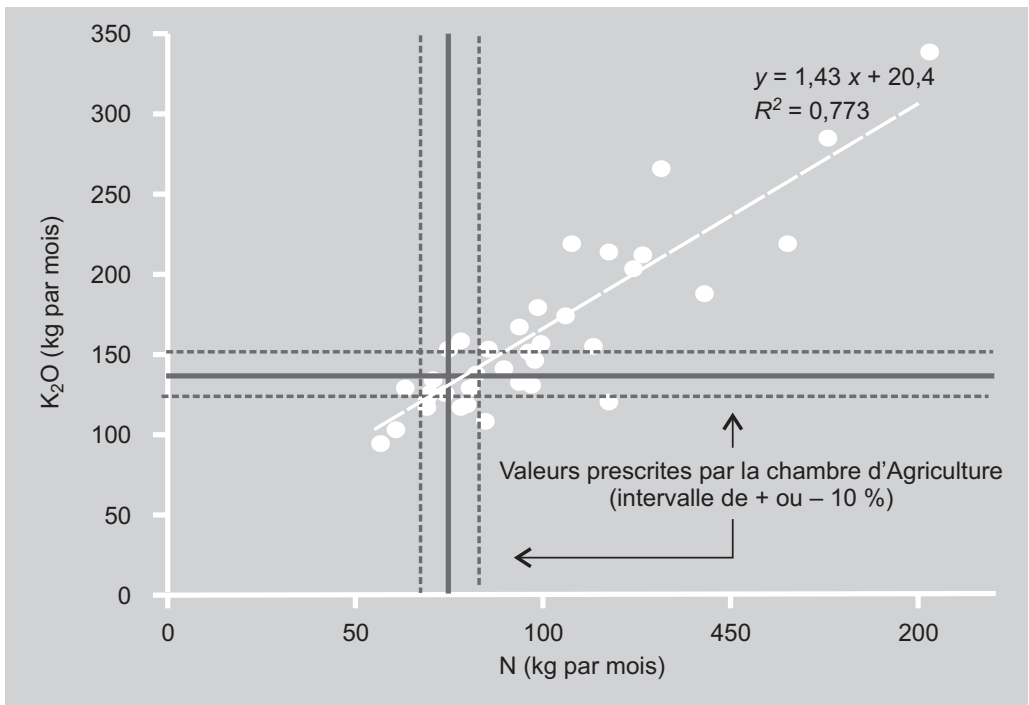

peuvent ou non consacrer à la culture. Cela permet de limiter les coûts et évite d'endommager les plants d'ananas.

- Un désherbage chimique généralisé effectué de façon "systématique " se fait " toutes les $x$ semaines " pour éviter le désherbage manuel et/ou les traitements lors du deuxième cycle.

Le plus souvent, l'utilisation d'herbicides est associée à du sarclage manuel. Sur ce poste, les pratiques mises en œuvre

Figure 1.

Apports mensuels de $\mathrm{K}_{2} \mathrm{O}$ et $\mathrm{N}$ appliqués par les producteurs d'ananas martiniquais selon des informations recueillies par enquêtes.

\section{Figure 2.}

Répartition des d'agriculteurs en fonction des pratiques de désherbage qu'ils appliquent en plantation d'ananas à la Martinique, selon des informations recueillies par enquêtes.

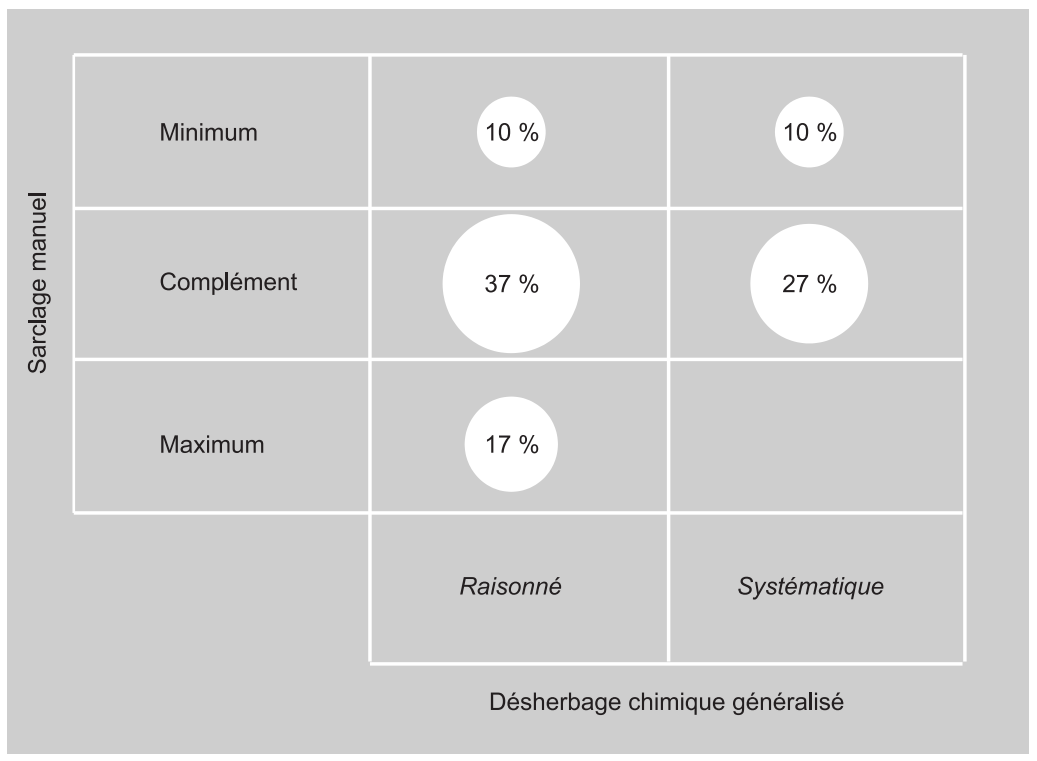


Figure 3.

Nombre d'applications d'herbicides effectuées pendant le premier cycle de production d'ananas, en fonction des exploitations étudiées en Martinique par enquêtes.

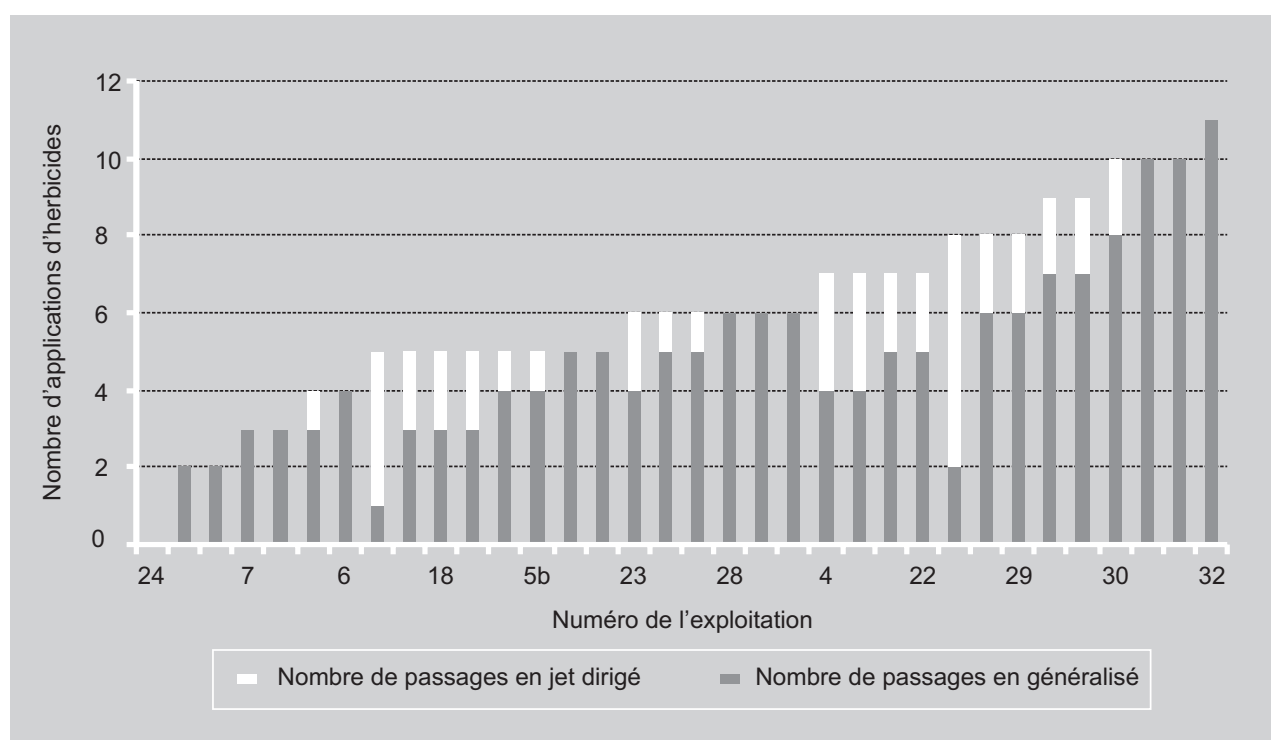

Le désherbage chimique peut être réalisé en généralisé sous forme de "pulvérisations " sur l'ananas ou en jet dirigé directement sur les " taches " d'herbes, à l'aide d'appareils à dos. Le nombre de traitements, en moyenne égal à six tous herbicides confondus, est assez variable (figure 3). Le désherbage chimique est peu pratiqué avant plantation et trop utilisé en cours de végétation.

Seuls $23 \%$ des planteurs réalisent un désherbage avant plantation, comme cela est généralement préconisé. C'est lors de ce désherbage que certains planteurs (10\%) emploient du bromacil, non homologué pour l'ananas. Même si l'utilisation de ce produit est tolérée avant plantation, l'impact environnemental de son utilisation peut être important car les exploitations qui l'utilisent représentent $53 \%$ de la surface cultivée en ananas. D'ailleurs, au cours des dernières années, le bromacil a été détecté pour neuf analyses d'eau réalisées sur la zone de production d'ananas, dont deux fois avec des quantités supérieures à la norme légale de $0,1 \mu \mathrm{g} \cdot \mathrm{L}^{-1}$.

En cours de végétation, les planteurs d'ananas utilisent en moyenne plus d'amétryne que ce qui est prescrit [moyenne générale entre (12 et 20$) \mathrm{L} \cdot \mathrm{ha}^{-1}$ pour une prescription de $\left.10 \mathrm{~L}^{\cdot} \mathrm{ha}^{-1}\right]$. La moitié des planteurs utilise au total plus de $12,5 \mathrm{~L} \cdot \mathrm{ha}^{-1}$ d'amétryne (figure 4 ). 
Pour le désherbage au diuron, les planteurs respectent généralement les recommandations : $67 \%$ des planteurs utilisent $4 \mathrm{~kg} \cdot \mathrm{ha}^{-1}$ ou moins de diuron, alors que la recommandation est égale à $5 \mathrm{~kg} \cdot \mathrm{ha}^{-1}$. Toutefois, $20 \%$ des planteurs appliquent des quantités supérieures au double de la quantité totale prescrite (figure 4).

Certains producteurs utilisent également de la cycloxydime (figure 5) ; cet herbicide n'est pas homologué pour la culture de l'ananas et il est appliqué le plus souvent en jet dirigé sur les chiendents.

L'analyse des pratiques des producteurs d'ananas vis-à-vis du désherbage a donc mis en évidence un recours aux produits de synthèse relativement important par rapport à une sarclage manuel peu utilisé ; $75 \%$ des producteurs d'ananas respectent les doses prescrites et au moins $50 \%$ d'entre eux utilisent des produits non homologués.

\subsubsection{Typologie des producteurs en fonction des pratiques culturales}

L'analyse des données recueillies au travers des questionnaires a permis d'établir une typologie des producteurs d'ananas à partir des itinéraires techniques pratiqués. Ainsi, l'analyse statistique de l'ensemble des variables jugées discriminantes pour chaque thème (préparation du sol, fertilisation, traitements phytosanitaires, production de rejets, etc.) a permis d'identifier, en fonction des pratiques agricoles, cinq grands types de producteurs d'ananas :

- Un premier type, que nous avons nommé "proche-références-plus ", concerne $20 \%$ des exploitations et $60 \%$ de la surface occupée par l'ananas en Martinique ; cette catégorie est celle qui applique au mieux les recommandations techniques de la Chambre d'agriculture :

- la fertilisation pratiquée est proche des recommandations : les doses d'application de $\mathrm{N}$ et $\mathrm{K}_{2} \mathrm{O}$ sont en dessous des recommandations ; le rapport $\left[\mathrm{K}_{2} \mathrm{O} / \mathrm{N}\right]$ est proche des recommandations ; les pulvérisations sont faites avec des doses croissantes jusqu'au TIF ;

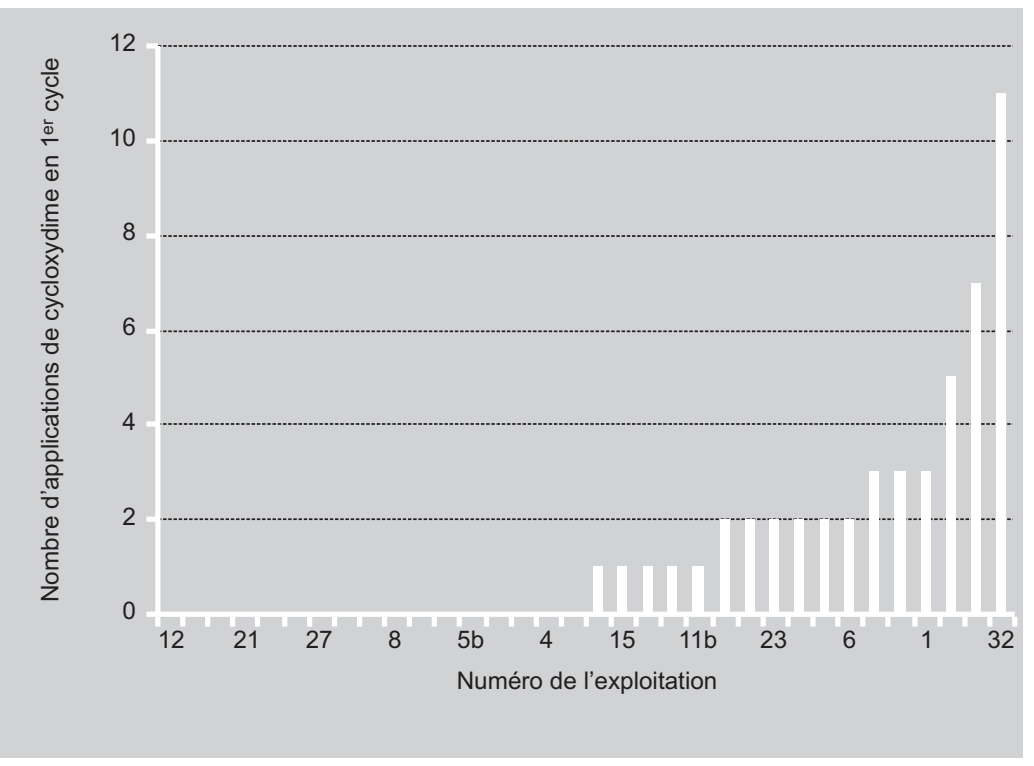

- le désherbage (chimique ou mécanique) est effectué avant plantation ;

- un désherbage raisonné se fait à doses moyennes en cours de végétation ;

- les pratiques phytosanitaires sont variables en ce qui concerne les nématicides : le Figure 5.

Nombre d'applications de cycloxydime effectuées pendant le premier cycle de production d'ananas, en fonction des exploitations étudiées en Martinique par enquêtes. terbufos, produit non homologué, est utilisé par une partie des planteurs ;

- les insecticides, parathion-méthyl et disulfoton, sont utilisés à des doses moyennes, inférieures aux recommandations ;

- le TIF se fait en un ou deux passages, mais avec uniquement de l'urée ;

- la production d'un rejet est conduite de diverses façons : en moyenne, une surfertilisation est appliquée, mais le rapport $\left[\mathrm{K}_{2} \mathrm{O} /\right.$ $\mathrm{N}]$ est plus proche des recommandations que celui pratiqué par les autres types de producteurs. Les doses de parathion-méthyl utilisées sont très inférieures aux recommandations ; il n'y a pas d'utilisation de disulfuton ou celle-ci est effectuée à des doses proches de celles prescrites. L'utilisation d'herbicides est très variable.

- Le deuxième type de producteurs d'ananas a été nommé " proche-références avec rejet "; il est propre à $27 \%$ des exploitations et $19 \%$ de la surface ; il applique un itinéraire technique proche des recommandations, 
excepté en ce qui concerne le rapport $\left[\mathrm{K}_{2} \mathrm{O} / \mathrm{N}\right]$, le mode d'application des engrais liquides et l'utilisation de nématicides :

- les doses d'apport en éléments fertilisants sont proches des recommandations, mais déséquilibrées car le rapport $\left[\mathrm{K}_{2} \mathrm{O} / \mathrm{N}\right]$ est trop faible et les pulvérisations se font à doses constantes entre la plantation et le TIF; la fumure de fond est systématique ;

- les produits de lutte contre les parasites sont appliqués avec des doses moyennes à fortes, avec de l'éthoprophos et du parathion-méthyl parmi les plus fortes doses, même si celles-ci restent toujours inférieures ou égales aux doses prescrites; parfois, le cadusaphos, produit non homologué mais dont une extension d'homologation devrait prochainement être formulée, est utilisé ;

- le désherbage chimique se fait seulement en cours de végétation avec des doses moyennes en herbicides ;

- le TIF est effectué avec des pratiques variables ;

- la sortie d'un rejet est assurée soit avec une faible fertilisation et sans autre produit phytosanitaire que le parathion-méthyl, soit avec une fertilisation fortement azotée et une utilisation de disulfoton, mais sans herbicides.

- Le troisième type de producteurs d'ananas identifié a été nommé " proche-références sans rejet "; il est présent pour $20 \%$ des exploitations et $5 \%$ de la surface ; son itinéraire technique est proche de celui du type 2 pour ce qui est de la fertilisation et du désherbage, mais il en diffère par l'utilisation des produits phytosanitaires ; ces planteurs ne suivent pas en général la production des rejets :

- la fertilisation est semblable au type 2 ;

- un désherbage chimique est effectué, mais seulement en cours de végétation et avec des doses modérées en herbicides, qui sont en moyenne inférieures aux recommandations ;

- l'utilisation des produits phytosanitaires est variable avec, en moyenne, plus de disulfoton et moins de parathion-méthyl et d'éthoprophos que préconisé ;
- le TIF est effectué en un ou deux passages, avec seulement de l'urée.

- Le quatrième type de producteurs d'ananas, ou "ferti-trop ", concerne $13 \%$ des exploitations et $9 \%$ de la surface ; la fertilisation est appliquée à trop fortes doses et elle est déséquilibrée, même lors de la sortie du rejet :

- la fertilisation diffère beaucoup des recommandations : les doses sont trop fortes, le rapport $\left[\mathrm{K}_{2} \mathrm{O} / \mathrm{N}\right]$ est trop faible et les pulvérisations d'engrais liquides se font à doses constantes entre la plantation et le TIF ;

- le désherbage chimique est effectué seulement en cours de végétation avec des doses moyennes en herbicides ;

- l'utilisation des produits phytosanitaires est variable ; en moyenne, les doses de disulfoton appliquées sont faibles et celles de parathion-méthyl sont modérées ;

- le TIF se fait en un seul passage ;

- la fumure de fond est raisonnée ;

- la sortie d'un rejet est assurée avec une fertilisation forte en azote, sans utilisation d'herbicides et avec des doses moyennes en produits phytosanitaires.

- Le cinquième type, nommé " phyto-trop ", gère $20 \%$ des exploitations et $7 \%$ de la surface ; il est caractérisé par une utilisation de fortes doses de produits phytosanitaires (insecticides et herbicides), y compris lors de la sortie du rejet :

- les désherbages chimiques sont fréquents et ils sont faits à fortes doses en cours de végétation ;

- les produits phytosanitaires sont utilisés à fortes doses : celles de parathion-méthyl et disulfoton sont supérieures aux recommandations ; les doses d'éthoprophos sont proches de celles préconisées par la Chambre d'agriculture ;

- la fertilisation est variable, parfois proche des recommandations et parfois éloignées ; en moyenne, les doses sont fortes ;

- la pratique du TIF est variable ;

- la fumure de fond se fait à $1 \mathrm{t} \cdot \mathrm{ha}^{-1}$ de chaux magnésienne ; 
- la sortie d'un rejet est assurée avec une fertilisation forte en azote, sans utilisation d'herbicides et avec de fortes doses de produits phytosanitaires.

La comparaison entre les pourcentages d'exploitations et de surfaces, liés aux pratiques de ces différents types de producteurs, traduit l'existence de différences structurelles fortes entre les plantations d'ananas en Martinique. Elle met en évidence le fait que les agriculteurs qui respectent le mieux les prescriptions techniques gèrent généralement de grandes exploitations. Cela peut s'expliquer par l'emploi de spécialistes, techniciens ou ingénieurs, par les grandes sociétés, ainsi que par l'adoption de modalités de gestion différentes.

L'intensification de la culture observée au début des années 90 [11] s'est poursuivie jusqu'en 2000 avec, pour conséquence, des déviations importantes par rapport aux recommandations officielles dans l'utilisation des intrants. Cette diversité de pratiques, parfois éloignées des recommandations [12, 13], aura des conséquences importantes sur les mesures à prendre pour la mise en place d'un système de management de la qualité dont les axes forts sont sécurité alimentaire, traçabilité et protection de l'environnement.

\subsection{Sécurité alimentaire et traçabilité}

La mise en ouvre du système de management de la qualité est une décision collective de la profession; elle est basée sur une démarche participative de l'ensemble des acteurs de la filière ananas.

\subsubsection{Management de la qualité des activités agricoles de la filière}

Le système de management de la qualité des activités agricoles mis en place par la Socomor repose sur un contrat de culture " ananas pour l'industrie " entre les producteurs et la coopérative. Ce contrat oblige les producteurs à respecter deux cahiers des charges mis au point par le comité de pilotage après une étude AMD (analyse et maîtrise des dangers pour la qualité du produit). Il oblige la coopérative Socomor à assurer des services de haut niveau, notamment la mise en place du système de management de la qualité et des actions à entreprendre sur le terrain, ainsi que la formation des producteurs. Ce contrat définit le mode de gestion des non conformités, les actions correctives à entreprendre et la gestion des déchets de production et de transformation.

- Le premier cahier des charges, ou cahier des charges "technique ananas industriel " (document interne Socomor), définit les exigences concernant le choix des parcelles et des techniques de production issues de l'AMD. Il définit également des recommandations techniques non contraignantes telles que le mode d'application des traitements ou l'équipement à utiliser. Il détermine les exigences en terme de traçabilité à l'échelle de la parcelle. Un guide de culture accompagne ce cahier des charges pour préciser les recommandations relatives aux pratiques culturales et pour permettre une homogénéisation des techniques de culture extrêmement variables d'un producteur à l'autre, comme l'avait montré l'état des lieux de la filière.

- Le second cahier des charges, ou cahier des charges "qualité ananas industriel " (document interne Socomor), est relatif à la qualité des fruits livrés à la coopérative pour la transformation. Il détermine les critères qualitatifs qui doivent être respectés et le mode d'agréage permettant de vérifier le respect de ces critères et de calculer les réfactions éventuelles.

L'ensemble de ces cahiers des charges est largement documenté selon le principe habituel d'un système de management de la qualité. Toutes les procédures sont décrites dans les documents internes de la coopérative. Toutes les opérations sont enregistrées. Des audits internes assurent non seulement le respect des cahiers des charges par les producteurs, mais ils permettent également une évaluation par les producteurs de la qualité des services et des prestations de la coopérative. Une formation permanente actualisant leurs connaissances en matière de management de la qualité permet une amélioration continue de la qualité de la production.

Enfin, des audits externes devraient permettre à terme d'obtenir un label ou une 
"indication géographique protégée " par rapport aux référentiels officiels autorisant une meilleure identification du produit martiniquais sur les marchés internationaux.

\subsubsection{Cahier des charges "technique ananas industriel "}

Le cahier des charges "technique ananas industriel " définit les exigences techniques à respecter par le producteur, ainsi que des recommandations applicables en fonction des situations individuelles des producteurs.

- Choix des parcelles

Le choix des parcelles doit être déterminé en fonction de critères tels que le relief, l'altitude, les caractéristiques du sol ou la localisation. Des recommandations sont précisées pour le choix de l'assolement et du précédent cultural pour éviter des contaminations éventuelles par des résidus de produits phytosanitaires interdits et respecter les cahiers des charges " clients spécifiques " (exemple : cas des produits destinés à l'alimentation des bébés).

- Suivi des parcelles

Après la caractérisation des parcelles, il faut assurer une traçabilité des opérations culturales grâce à l'utilisation d'une fiche parcellaire.

- Pratiques culturales

Dans le cahier des charges "technique ananas industriel", des exigences strictes sont décrites concernant la préparation du sol (déclaration des dates), les densités et l'origine des plants, les amendements et la fertilisation en ce qui concerne le respect des exigences réglementaires européennes et françaises, le délai de traitements avant TIF, ainsi que les doses à déclarer sur la fiche parcellaire. Ces exigences sont assorties de recommandations permettant une certaine souplesse dans l'élaboration de l'itinéraire technique. Elles se retrouvent dans le guide de culture.

- Traitements phytosanitaires

Pour les traitements phytosanitaires, les exigences concernent le respect des règlements communautaires et la déclaration des traitements sur la fiche parcellaire. Outre les méthodes d'application et le matériel utilisé, le recours à des analyses de résidus réalisées par la Socomor est recommandé.

\section{- Activités lors de la récolte}

Les producteurs doivent préparer les fruits avant livraison à l'usine en retirant la couronne. Des délais optimaux entre la récolte et la livraison sont imposés. Le mode de transport est également une exigence quant à l'identification des lots, la hauteur de stockage dans les bennes et les soins apportés aux fruits.

\subsubsection{Cahier des charges "qualité ananas industriel "}

Le cahier des charges "qualité ananas industriel " définit les principaux défauts des fruits tels qu'ils peuvent être libellés soit dans le Codex Alimentarius, soit dans des ouvrages spécifiques sur la qualité de l'ananas réalisés pour d'autres filières ananas [14]. Dans ce document, sont mentionnés tous les défauts externes (blessures, coups de soleil, choc ou écrasement, déformations, salissures des fruits stockés) ou internes [maladies fongiques (pourriture molle à Ceratocystis sp. ou taches noires à Penicillium f.), brunissements internes, translucidité (fruits vitreux)] pouvant avoir un impact sur la qualité et la quantité finale du produit transformé.

Le mode d'agréage déterminé en accord avec les producteurs est également décrit dans ce cahier des charges. Les défauts sont observés sur un échantillon brut, puis sur les fruits " cylindrés". Une partie des fruits peut être purement et simplement rejetée. Des fruits présentant de légers défauts peuvent être agréés partiellement. L'ensemble conduit à définir un pourcentage de réfaction sur le tonnage brut livré.

Enfin, le cahier des charges " qualité ananas industriel " fait référence au Guide des bonnes pratiques bygiéniques du JO, juillet 1999, et à la Directive européenne 93-43.

\subsubsection{La traçabilité parcellaire}

Les recommandations concernant les pratiques culturales existent et pourtant des déviations importantes ont été observées 
dans l'analyse de ces pratiques. Il apparaît nécessaire de mettre en place un système de traçabilité interne à la filière qui permettra de suivre, parcelle par parcelle, la mise en œuvre des recommandations techniques; ce système de traçabilité devrait permettre aussi, dans une certaine mesure, un affichage, pour les clients de la Socomor, de pratiques respectueuses de principes aujourd'hui incontournables de sécurité alimentaire et de respect de l'environnement.

La traçabilité parcellaire est l'un des éléments important de ce système de management de la qualité pour lequel la recherche a pu apporter une contribution importante à travers la réalisation d'une base de données simple d'utilisation "MARTAnanas", relative aux producteurs et à leurs parcelles. Cette base est gérée par la coopérative Socomor.

Les éléments pris en compte pour cette traçabilité sont la parcelle, la prévision des opérations culturales, la diffusion de fiches mensuelles de prévision des opérations culturales producteur par producteur, la production de fiches de traçabilité complètes, ainsi que l'enregistrement des données concernant les livraisons de fruits.

- La parcelle, unité de base de production, est située dans le contexte "filière, producteur, plantation ". Elle est créée dans la base de données à partir d'une déclaration du producteur précisant sa localisation exacte, sa date de plantation, son nombre de plants, son itinéraire technique. La Socomor utilise un système d'information géographique (SIG), notamment pour les déclarations de surfaces plantées faites à l'administration (Direction de l'agriculture et de la forêt).

- Pour la prévision des opérations culturales depuis la plantation jusqu'à la destruction des plants, la base de données déduit, de la date de plantation, une date probable de traitement d'induction florale (TIF), toutes les opérations culturales y compris l'application d'intrants, l'utilisation des pesticides et les besoins en eau. Elle donne une date de récolte probable en fonction de l'itinéraire technique recommandé pour le producteur concerné, de la date de plantation et des relevés de températures quotidiens permettant d'évaluer l'intervalle entre le TIF et la récolte en calculant la somme des températures correspondantes pour une zone donnée (adapté du logiciel "Aloha pineapple " $([9,10]$ et Touron J., Fournier P., commun. pers., 2000). La base de données prévoit aussi un tonnage récolté en fonction des cycles précédents cultivés sur la même parcelle et de coefficients de correction prenant en compte la croissance végétative pour le cycle en cours.

- Des fiches mensuelles de prévision des opérations culturales producteur par producteur sont également utilisées pour l'enregistrement des données réelles par le producteur ; elles permettent une actualisation mensuelle de la base de données.

- Des fiches complètes de traçabilité, parcelle par parcelle, présentant l'ensemble des opérations et évènements ayant eu lieu ainsi qu'un bilan minéral de la fertilisation sont produites.

- L'enregistrement des données concernant les livraisons de fruits via une connexion avec le logiciel de pesée de la coopérative permet d'avoir un tableau comparatif des flux prévisionnel et réel entrant à l'usine. Il s'ensuit une meilleure gestion des opérations de transformation (tonnages à traiter quotidiennement et personnel requis).

Des éléments additionnels, comme un calcul des besoins en intrants de l'ensemble de la filière, permettent une gestion plus efficace de la centrale d'achat de la coopérative.

\subsection{Nouvelles variétés}

Actuellement, la culture de l'ananas en Martinique repose sur l'exploitation d'une seule variété, la Cayenne lisse, et l'essentiel de ses débouchés est la transformation par la Socomor. Cependant, un facteur déterminant pour la pérennité de la filière ananas serait sa capacité de diversification qui, outre la transformation, s'appuierait sur le développement d'une production de fruits frais destinés aux marchés locaux ou d'exportation. De plus, le développement de variétés innovantes pourrait accroître la compétitivité de la production martiniquaise, quant à la qualité intrinsèque du produit et à la réduction des impacts environnementaux. Dans ce 
Figure 6.

Présentation des fruits de trois hybrides d'ananas sélectionnés par le Cirad et retenus pour un développement en Martinique (industrie et/ou fruit frais) .
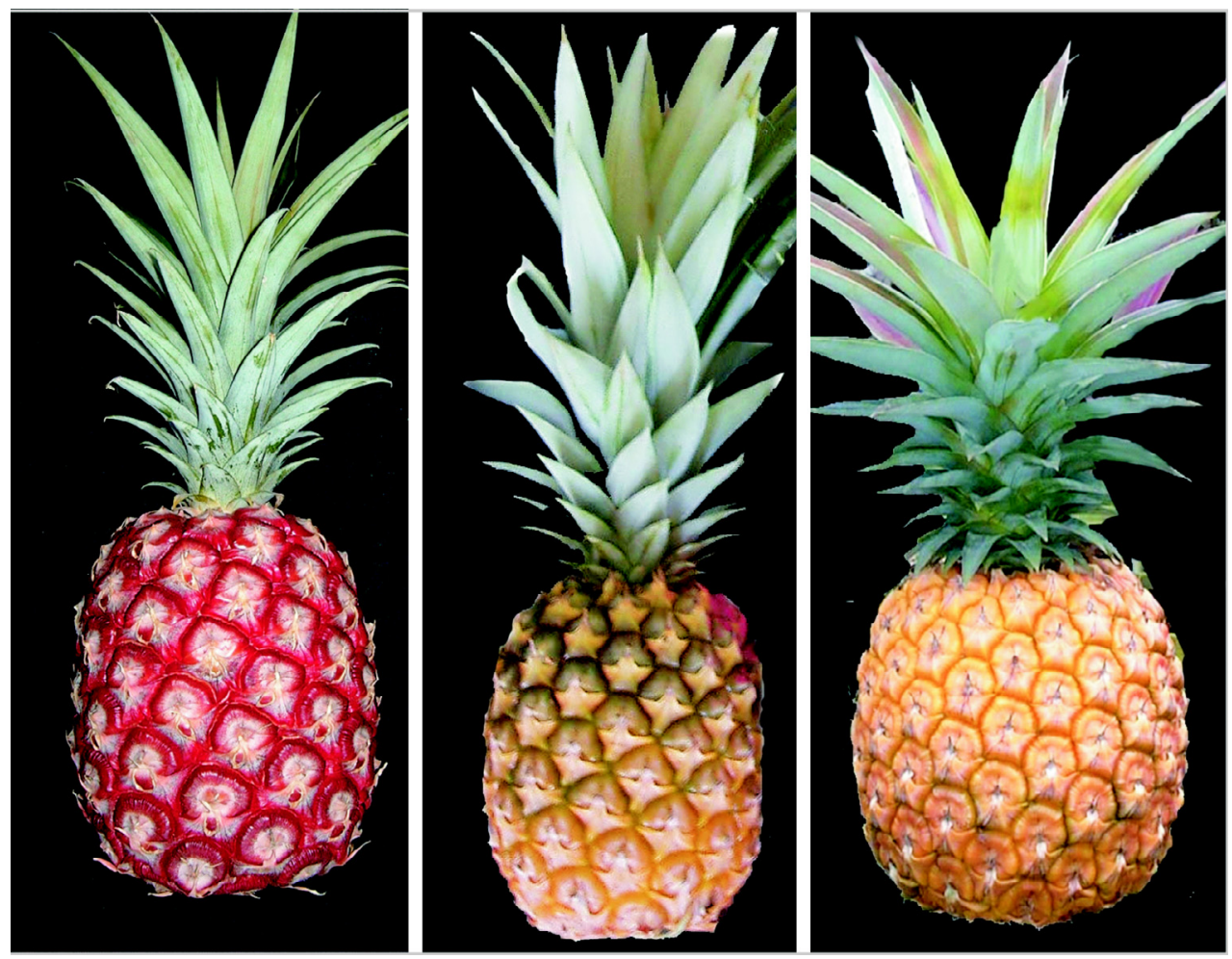

cadre, les recherches menées par le Cirad permettent de proposer de nouvelles variétés mieux adaptées aux marchés ; la qualité de leur fruit est alors améliorée pour la consommation en frais locale, voire pour l'exportation ; ces variétés peuvent également être propices aux processus de transformation car elles permettent une augmentation des rendements.

Ce sont des sélections clonales issues du matériel collecté au cours des missions de prospection du bassin amazonien au cours des années 80 à 90 [15] ou des variétés hybrides créées dans le cadre d'un programme d'amélioration génétique [16].

\subsubsection{Sélections clonales}

Au sein des clones de type Cayenne collectés au cours de prospection, le Cirad a sélectionné deux clones originaires de Guyane française (GF090 et GF449), qui, conservant les qualités intrinsèques du clone cultivé en Martinique (Champaka), présentent un rapport [sucre / acidité] plus élevé (respec- tivement 1,45 et 1,35 contre 1,06 pour Champaka). Ces deux clones pourraient avantageusement se substituer au type local tant pour la transformation que pour la consommation en frais.

\subsubsection{Variétés hybrides}

Les hybrides proposés dans le cadre d'une reconversion variétale martiniquaise sont issus d'un vaste programme de croisements faisant intervenir le Cayenne lisse et les variétés colombiennes Péroléra et Manzana qui est un clone rouge orangé de Péroléra. Le programme d'amélioration a eu pour objectif d'ajouter aux qualités du Cayenne des caractères propres aux Péroléra et Manzana : une forte teneur en acide ascorbique, une chair jaune d'or plus ferme, moins juteuse et peu fibreuse et une forme très cylindrique du fruit [16].

Après de nombreux cycles d'évaluation et de sélection au sein des milliers d'hybrides produits, trois hybrides ont été retenus : Flhoran 41, Flhoran 53 et Flhoran 55 
(figure 0 ). Les fruits sont d'un poids similaire à Cayenne lisse (1,3 à 1,9 kg). Les qualités organoleptiques ont été améliorées avec une teneur en sucre plus élevée que chez Cayenne pour une acidité comparable. Ces hybrides ont également hérité d'une forte teneur en acide ascorbique de (14 à 18) $\mathrm{mg} \cdot 100 \mathrm{~mL}^{-1}$, soit deux à trois fois plus élevée que celle des fruits du Cayenne. La pulpe présente les caractéristiques recherchées de fermeté et de couleur (aspect visuel plus attrayant), conduisant, notamment pour la transformation, à une amélioration significative de la tenue à la découpe (figure 7).

Par ailleurs, les fruits de Flhoran 41 et Flhoran 53 présentent une forme cylindrique très marquée les rendant parfaitement adaptés au cylindrage en usine. Enfin, à maturité, le fruit de Flhoran 41 se colore naturellement en rouge orangé, caractère d'autant plus marqué que les températures sont fraîches. Cette caractéristique visuelle, très attractive, le démarque clairement aux yeux des consommateurs des autres variétés du marché.

Flhoran 53 semble être l'hybride le mieux adapté à la transformation et devrait permettre une augmentation significative des rendements en usine. Flhoran 41 est un produit mixte permettant aussi bien la transformation en usine que la vente en frais : les rendements en cubes obtenus en tests usine sont de $63,3 \%$ pour Flhoran 41 contre seulement (30 à 40)\% pour les fruits de Cayenne lisse ; sur le marché du frais, les qualités organoleptiques et visuelles de cet hybride pourront contribuer à une augmentation de la qualité des produits mis en marché.

Le fruit de Flhoran 55 est de forme globulaire, ce qui le rend impropre à la transformation en tranches ou en cubes. En revanche, son taux record de sucre (près de $18^{\circ}$ Brix, avec un rapport [sucre / acidité] de 1,6) en fait une variété très intéressante pour le marché du frais. Cet hybride est cependant handicapé par une production de rejet faible et tardive, compromettante pour son développement.

À terme, le développement de nouvelles variétés hybrides résistantes aux ravageurs et parasites pourrait contribuer à la réduc-

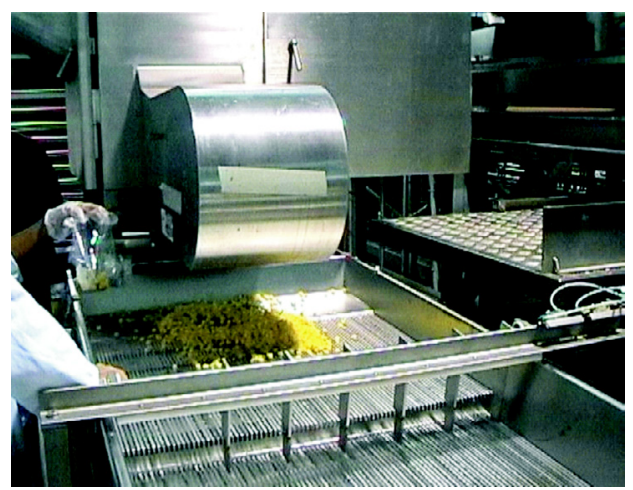

Figure 7.

Transformation en cube de l'ananas (coopérative Socomor, Martinique). tion des nuisances environnementales. En particulier, la création de variétés tolérantes aux nématodes répondrait à la double nécessité d'une meilleure préservation du milieu et viendrait pallier l'absence de nématicides homologués, selon la législation européenne, en culture d'ananas.

\section{Conclusion}

La décision de restructurer la filière ananas de Martinique résulte avant tout de considérations économiques : la compétitivité de cette filière est faible devant les produits industriels à bas coûts des producteurs asiatiques. La profession a longtemps été soutenue grâce aux aides nationales et européennes, car les coûts élevés de production en Martinique sont en grande partie liés aux charges salariales que supportent les producteurs face à des producteurs d'autres pays non soumis aux mêmes contraintes. Ces aides s'amenuisant d'année en année, la survie de la filière passe par des innovations techniques (nouveaux produits, nouvelles variétés), mais aussi la mise en avant d'une spécificité qui préconise une production respectueuse de la sécurité alimentaire et de la protection de l'environnement.

Des recommandations techniques existent déjà, mais une partie non négligeable des agriculteurs (environ 1/3) met en œuvre des pratiques pouvant avoir des conséquences néfastes sur l'environnement et sur la qualité du fruit et, donc, potentiellement, sur la santé humaine. Une homogénéisation des pratiques se rapprochant de recommandations visant des objectifs de qualité est 
nécessaire ; celles-ci pourraient être rassemblées dans un guide de culture adapté. Cette démarche a conduit au développement d'un programme d'assurance qualité avec instauration d'un système de traçabilité parcellaire, destiné à garantir la sécurité alimentaire.

La mise au point de nouvelles variétés mieux adaptées aux marchés et aux nouveaux procédés de transformation devrait permettre dans un premier temps d'améliorer la compétitivité de la filière grâce à une reconversion variétale partielle. La mise au point de nouveaux itinéraires techniques plus respectueux des exigences de protection de l'environnement pour ces mêmes variétés devrait faciliter dans un deuxième temps la mise en ouvre d'un système de management de la qualité. Enfin, à plus long terme, la création de variétés résistantes ou tolérantes aux principaux parasites de l'ananas à intégrer dans un système de culture spécifique permettra de réduire au strict minimum l'impact d'intrants dangereux pour le milieu.

\section{Références}

[1] Anon., Système de management de la qualité de la production agricole, AFNOR, NF V 01005, 2000, Agri Confiance ${ }^{\circledR}, 14$ p.

[2] Capillon A., Sebillote M., Étude des systèmes de production des exploitations agricoles. Une typologie, in: Servant J., Pinchinat A. (Eds.), Caribbean seminar on farming systems research methodology, Pointe à Pitre, Guadeloupe, FWI, May 4-8, 1980, IICAINRA, San Jose, Costa Rica, 1982, 85-111.

[3] Bonneviale J.R., Jussiau R., Marshall E., Approche globale de l'exploitation agricole. Comprendre le fonctionnement de l'exploitation agricole : une méthode pour la formation et le développement, INRAP, document $n^{\circ}$ 90, Dijon, France, 1989, 329 p.

[4] Dobremez L., Bousset J.-P., Rendre compte de la diversité des exploitations agricoles: une démarche d'analyse par exploration conjointe de sources statistiques, comptables et technico-économiques, Cemagref éditions, Coll. Études du Cemagref, gestion des territoires, n 17, St-Martin d'Hères, France, 1996.

[5] Teissier J.H., Relations entre techniques et pratiques. Conséquences pour la formation et la recherche, ENSSAA, bulletin INRAP $\mathrm{n}^{\circ} 38$, Dijon, France, 1979, $13 \mathrm{p}$.

[6] Testut M., Analyse des pratiques et stratégies des producteurs d'ananas de la Martinique, Cemagref, rapport, Fort de France, Martinique, 2001.

[7] Milleville P., Recherches sur les pratiques des agriculteurs, Cah. Rech. Dév. 16 (1987) 3-7.

[8] Labe V., Palm R., Statistique, empirique, informelle: quelle enquête pour la collecte d'informations sur les exploitations agricoles? Cah. Agric. 8 (5) (1999) 397-404.

[9] Malézieux É., Zhang J., Sinclair É., Bartholomew D., Predicting pineapple harvest date in different environments using a computer simulation model, Agron. J. 86 (4) (1994) 609-617.

[10] Sananikone T., Simulation de la température interne du fruit de l'ananas, INA-PG, Mém. DAA, Grignon, France, 1995.

[11] Marie-Alphonsine P., Marie F., Moutoussamy G., Intensification et développement de la culture de l'ananas : cas des petits planteurs de Martinique, in: Barbeau G., Ramnanan N., Walmsley D. (Eds.), Proc. Reg. Workshop Pineapple Martinique, March 22-24, 1994, IICA, Port of Spain, Trinidad and Tobago, 79-83.

[12] Py C., Lacoeuilhe J.-J., Teisson C., L'ananas, sa culture, ses produits, G.-P. Maisonneuve et Larose, Paris, France, 1984, 498 p.

[13] Anon., Ananas de Martinique, de la plantation à la récolte. Formation des personnels. Outils pédagogiques, Fonds National d'Assurance Formation des Salariés des Exploitations et Entreprises Agricoles (Fafsea), Pantin, France, 1999, $32 \mathrm{p}$.

[14] Soler A., Ananas, critères de qualité, Cirad Coleacp, Anduze, France, 1992, 48 p.

[15] Duval M.F., Coppens d'Eeckenbrugge G., Ferreira F.R., Bianchetti L.D.B., Cabral J.R.S., First results from joint EMBRAPA-CIRAD ananas germplasm collecting in Brazil and French Guyana, in: Martin-Prevel P., Hugon R. (Eds.), Proc. II Int. Pineapple Symp., Acta Hortic. 425 (1997) 137-144.

[16] Marie F., Coppens d'Eeckenbrugge G., Bernasconi B., Pineapple breeding at CIRAD. I. Evaluation and selection of 'Smooth Cayenne' $\times$ 'Manzana' hybrids, in: Subhadrabandhu S., Chairidchai P. (Eds.), Proc. III Int. Pineapple Symp., Acta Hortic. 529 (1998) 147-153. 


\section{Manejo de la calidad en el industria piñera en Martinica.}

Resumen - Introducción. La industria piñera de Martinica se está reorganizando profundamente como lo hicieron otros sectores de alimentación humana par cumplir con los nuevos requisitos de seguridad alimenticia y de protección del environamiento : Manejo de calidad a todos los niveles de la industria y introducción de nuevas variedades de piña. Un análisis de las técnicas agrícolas usuales de los productores permitió a la autores de proponer unos cambios necesarios así que un nuevo sistema de producción. Material y métodos. Una topología de las fincas ha sido elaborada a través del análisis de las técnicas agrícolas usuales y de las estrategias de los productores. Un comité piloto incluyendo representantes de la mayor parte de los actores de la industria, propone un sistema de manejo de la calidad refiriéndose a las normas Agriconfiance ${ }^{\circledR}$. Nuevas variedades de piña han sido evaluadas para transformación o para el mercado fresco. Resultados y discusión. Se notan diferencias variables y generalmente amplias entre las recomendaciones y los usos reales: demasiado insumos, sea fertilizantes, sea pesticidas o los dos. El sistema de manejo de calidad está basado en un contrato entre la cooperativa y el productor. Las reglas se encuentran en dos códigos de prácticas, uno para la producción de la fruta en el campo y el otro para la transformación. Un sistema de trazabilidad ha sido elaborado permitiendo el registro y previsión al nivel de la parcela. Nuevas variedades de piñas fueron utilizadas para transformación dando mejores resultados que la variedad Cayena lisa. Conclusión. La reorganización de la industria piñera en Martinica resulta de problemas económicos. Esta implementación necesitó el desarrollo de un programa global de seguridad de calidad con un sistema de trazabilidad al nivel de parcela para garantizar la seguridad alimenticia. Una homogeneización de los usos es necesaria, refiriéndose a recomendaciones técnicas para mejorar la calidad. Podrían ser agregadas a una guía técnica específica. El uso de nuevas variedades con mejor rendimientos de transformación será también un elemento importante del suceso de la reorganización.

Martinico / Ananas comosus / manejo del cultivo / seguridad alimentaría / protección ambiental / ensayos de variedades / frutas / calidad 